\title{
EFECTIVIDAD DEL EJERCICIO Y LA EDUCACIÓN TERAPÉUTICA EN PACIENTES CON DOLOR CRÓNICO: UNA REVISIÓN BIBLIOGRÁFICA
}

\author{
Vicente Muñoz-Alarcos, PT ${ }^{1}$; Maria del Mar Ivars Crespo, MSc²; Luis Suso-Martí, PhD 1,3*, Aida Herranz-Gómez, MSc ${ }^{3}$; \\ Patricio Alba-Quesada, PT ${ }^{1}$; Ferran Cuenca-Martínez, PhD ${ }^{3}$; Arturo Such-Sanz, PT \\ 1. Departamento de Fisioterapia y Enfermería, Universidad CEU Cardenal Herrera, CEU Universities, Valencia, España. \\ 2. Hospital Universitario y Politécnico La Fe, Valencia, España. \\ 3. Motion in Brains Research Group, Institute of Neuroscience and Sciences of the Movement (INCIMOV). Centro Superior de Estudios Universitarios La \\ Salle. Universidad Autónoma de Madrid. 28023 Madrid (España).
}

\section{Correspondencia:}

Luis Suso-Martí, PhD Department of Physiotherapy, Universidad CEU Cardenal Herrera, CEU Universities,

Valencia, Spain. Teléfono: 961369000 E-mail: 1uis.suso@gmail.com

\section{Conflicto de Intereses:}

Los autores declaran no tener ningún conflicto de intereses

Financiación:

Este estudio no ha recibido ninguna subvención específica de organismos de financiación del sector público, comercial o sin ánimo de lucro.

\section{DOI:}

htpps://doi.org/10.37382/jomts.v3i1.470

\section{Recepción del Manuscrito:}

09-Julio-2021

\section{Aceptación del Manuscrito:}

21-Julio-2021

\section{RESUMEN}

Objetivo: El objetivo de esta revisión bibliográfica fue evaluar el efecto de la educación neurofisiológica en dolor en combinación con ejercicio terapéutico sobre la intensidad del dolor y los factores psicosociales en pacientes con dolor crónico.

Métodos: Se realizó una búsqueda en las bases de datos PubMed, Web of Science, CINAHL y SCOPUS. Se emplearon diferentes términos médicos como estrategia para encontrar estudios que aborden la educación neurocientífica y el ejercicio. La escala PEDro se utilizó para evaluar la calidad metodológica de los estudios.

Resultados: Se obtuvieron un total de seis estudios. Los resultados muestran diferencias significativas entre el tratamiento control y el tratamiento experimental a favor de este último, tanto en la intensidad de dolor como en las variables psicosociales analizadas, especialmente en la kinesiofobia.

Conclusiones: La terapia conjunta de educación en neurofisiología del dolor y el ejercicio terapéutico produce una mejora en las variables de ámbito psicosocial y la intensidad de dolor en pacientes con dolor crónico.

Palabras clave: Sensibilización central, Biopsicosocial, Neurociencia, Ejercicio cognitivo
Licensed under: CC BY-NC-SA 4.0

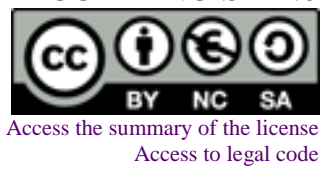




\section{INTRODUCCIÓN}

El dolor tradicionalmente ha sido clasificado según el tiempo de evolución, dividiéndose en agudo, subagudo o crónico. El dolor se considera crónico cuando alcanza o supera los tres meses de evolución dentro de un proceso normal de curación (Raja et al., 2020). Desde el punto de vista del paciente, la percepción continuada de dolor puede desembocar en alteraciones tanto psicológicas como funcionales, influenciando de forma negativa en el desarrollo normal de la vida cotidiana (Pergolizzi et al., 2013).

El modelo biomédico parece no ser capaz de dar una explicación plena sobre la complejidad del dolor crónico, ya que la prevalencia de dolor en la población general es amplia y en aumento (Langley, 2011). Los tratamientos que normalmente se emplean desde este modelo, como son la terapia manual, el uso de agentes físicos y las pautas generales de actividad física, no han mostrado resultados positivos con una significancia considerable (Cuenca-Martínez et al., 2018).

Desde este paradigma, el modelo biopsicosocial emerge para reinterpretar el significado de dolor crónico y conceptualizarlo dentro de un proceso de sensibilización central del sistema nervioso ( $\mathrm{SN}$ ), que figura como el causante principal de una respuesta neural hiperexcitable que lleva a procesos compatibles con la sensibilización central (Roussel et al., 2013).

En este contexto, se producen una serie de disfunciones del sistema nervioso central (SNC) que incluyen la alteración del procesamiento sensorial y discriminativo del cerebro, el mal funcionamiento de las vías nociceptivas facilitadoras e inhibitorias, los cambios morfológicos de la materia gris del cerebro y la afección psicosocial que agrava el cuadro clínico (Moseley and Flor, 2012; Turk et al., 2016; Adams and Turk, 2018).

Con el modelo biopsicosocial aparece la neurociencia como tratamiento mediante la educación neurocientífica en el dolor y el ejercicio terapéutico. Por una parte, la educación en neurociencia del dolor se centra en disminuir la sensación de amenaza y cambiar las creencias del dolor en el paciente (Louw et al., 2011). Por otra parte, el uso del ejercicio terapéutico es una de las primeras opciones dentro de un tratamiento para reducir el dolor y, los estudios señalan los cambios a nivel central que se producen como posible explicación de su eficacia (Koltyn et al., 2014; Booth et al., 2017). Sin embargo, algunos estudios muestran discrepancias, y no está totalmente claro si el ejercicio puede actuar produciendo una respuesta de hipoalgesia o hiperalgesia. En este sentido, se ha sugerido que el ejercicio puede suponer un estrés o amenaza para el paciente con dolor crónico, y la interpretación del mismo puede influir la respuesta hipoalgésica producida (Ceballos-Laita et al., 2020; Vaegter and Jones, 2020). Se hipotetiza que la educación en neurociencia del dolor podría reducir los factores psicosociales en los pacientes con dolor crónico, produciendo una reinterpretación del movimiento y el ejercicio, mejorando así las respuestas hipoalgésicas del mismo. Por lo tanto, el objetivo de esta revisión bibliográfica fue conocer el efecto de la combinación de la educación neurocientífica en el dolor y el ejercicio terapéutico en el paciente con dolor crónico.

\section{MÉTODOS}

\section{Diseño del estudio}

El presente trabajo consiste en una revisión sistemática. El trabajo se realizó de acuerdo con las directrices del "Preferred Reporting Items for Systematic Reviews and Meta-analysis" (PRISMA). El modelo PRISMA se compone de una lista de verificación de 27 ítems y un diagrama de flujo de cuatro fases, el cual ayuda a informar sobre las revisiones sistemáticas y los metaanálisis (Liberati et al., 2009).

\section{Estrategia de búsqueda}

Las bases de datos seleccionadas para la búsqueda de recursos científicos fueron: PubMed, Web of Science, CINAHL y SCOPUS. Se emplearon términos médicos (MeSh) y las palabras clave en inglés con las siguientes connotaciones: musculoeskeletal chronic pain; pain sensizitation; central pain sensizitation; dual task; neuroscience education; physical therapy; cognitive training; control motor; behavioral approach; exercise therapy. 


\section{Criterios de selección y exclusión}

Los criterios de inclusión que se aplicaron en la selección de los documentos se basaron en factores metodológicos y clínicos, como los criterios de población, intervención, control, resultados y diseño del estudio (método PICO) (Stone, 2002).

Población: Sujetos con dolor crónico

Intervención: Abordaje bioconductual basado en ejercicio terapéutico y educación terapéutica.

Comparación: Tratamiento habitual.

Variables de resultado: Intensidad de dolor, discapacidad o factores psicosociales.

Se incluyeron todos los artículos en idioma inglés o español, sin limitación temporal en su publicación.

\section{Evaluación de la calidad metodológica}

La evaluación e la calidad metodológica se realizó mediante la escala PEDro. Los criterios metodológicos se calificaron: si el criterio se cumplía (1 punto) o si no se cumplía (0 puntos), siendo la puntuación máxima posible de 10 puntos. Se considera una calidad aceptable si se cumplen 6 o más criterios(Verhagen et al., 1998; Maher et al., 2003).

\section{RESULTADOS}

De una búsqueda inicial donde se encontraron 65 artículos, finalmente tras aplicar los criterios de selección quedaron elegidos 6 ensayos clínicos aleatorizados. El diagrama de flujo de búsqueda y selección de los estudios se muestra en la Figura 1.

Las características principales de las que se extrajeron los datos de los estudios incluidos en la presente revisión sistemática se muestran en la Tabla 1.

\section{Calidad metodológica}

La calidad metodológica de los estudios incluidos fue buena, con puntuaciones entre 6 y 7 puntos (Tabla 2).

\section{Características de los tratamientos}

Los artículos estudiaron principalmente los efectos de la educación en el dolor basada en neurociencia en combinación con ejercicio terapéutico. Dos artículos compararon, además, si la terapia manual tenía un efecto superior sobre la combinación de educación y ejercicio.

Figura 1. Diagrama de flujo de los artículos seleccionados con el método PRISMA.

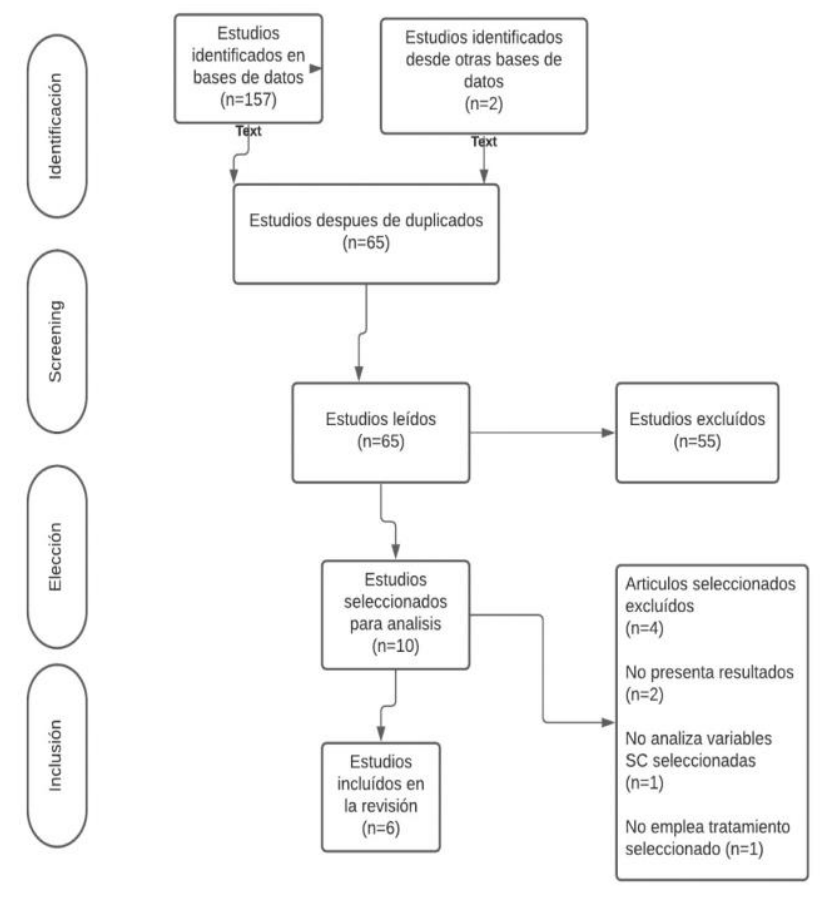

Aunque todos los artículos analizados compartían las bases de educación neurofisiológica, las estrategias de ejercicio terapéutico fueron diferentes entre ellos: tres de los artículos incentivaron el trabajo cognitivo dentro de sus sesiones de ejercicio terapéutico o ejercicios graduales hacia el gesto doloroso, un artículo analizó dentro del programa de ejercicio técnicas de movilización neural y el resto de artículos basaron sus programas en ejercicios convencionales de control motor relativos al área de estudio.

Los tratamientos experimentales se compararon con diferentes tratamientos control, siendo el ejercicio general (fortalecimiento, estiramientos, trabajo aeróbico) el tratamiento más utilizado, seguido de la terapia manual ortopédica (movilizaciones accesorias postero-anteriores), los agentes físicos (termoterapia y electroterapia) y la educación biomecánica/biomédica. 


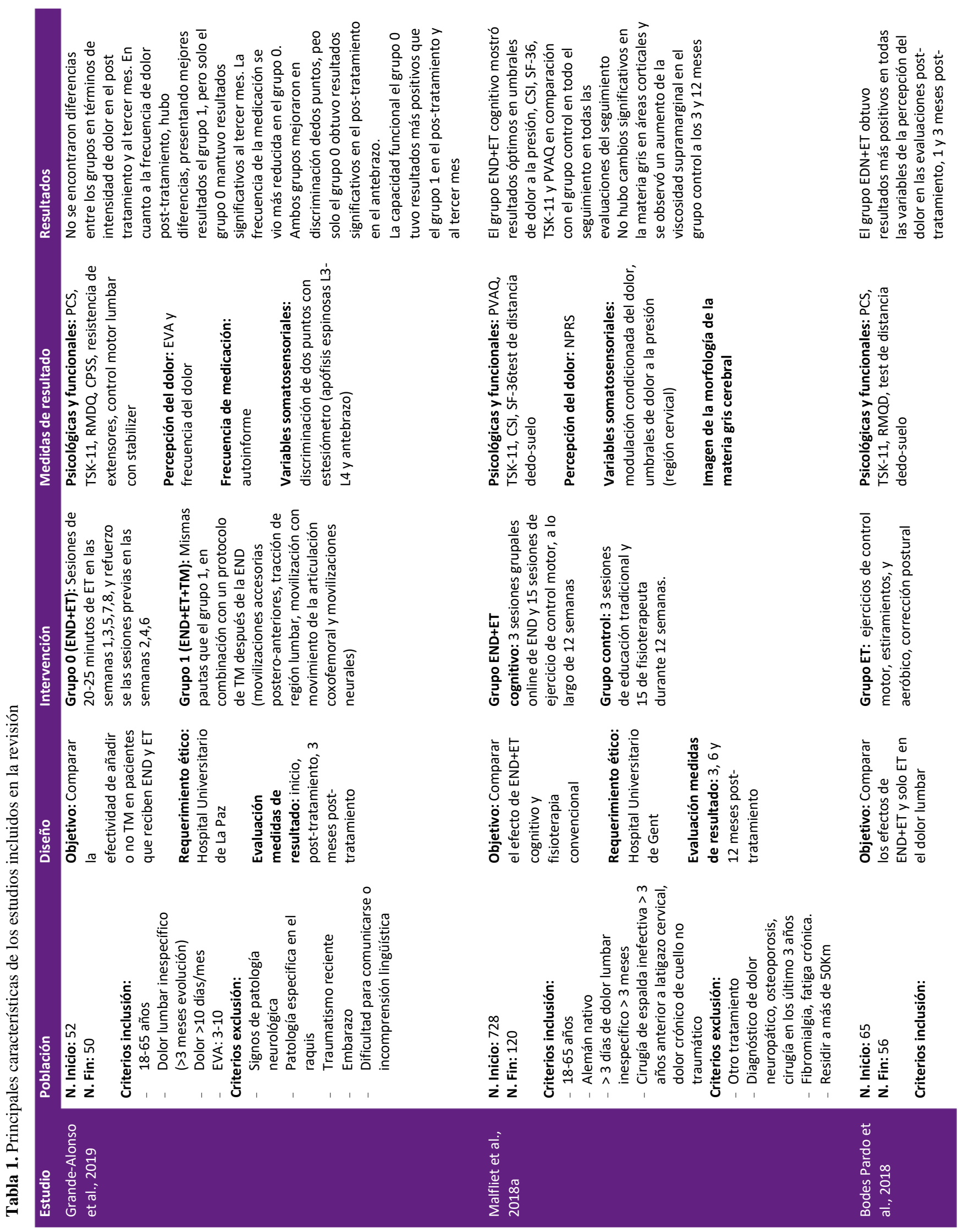



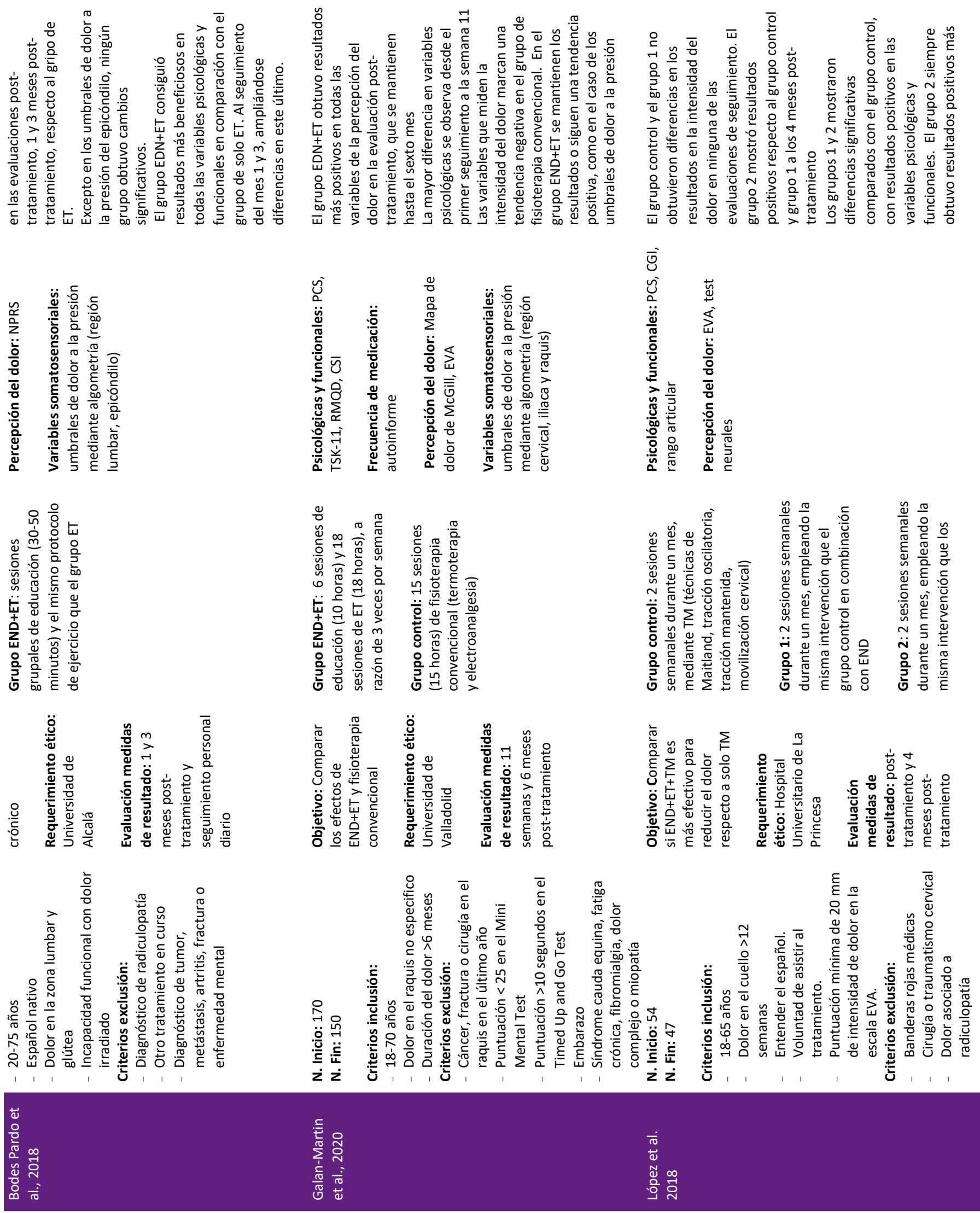


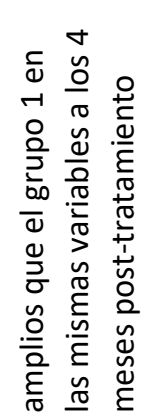

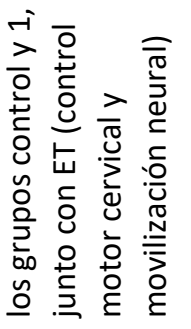

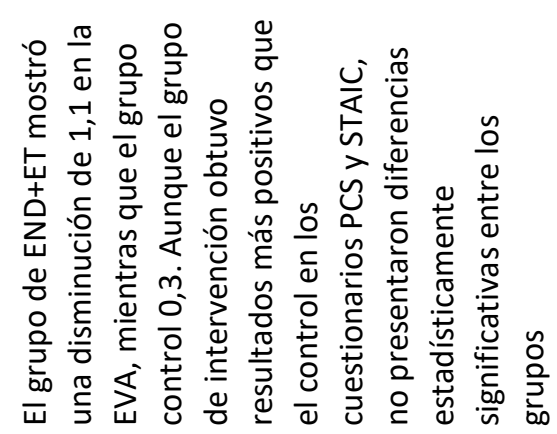

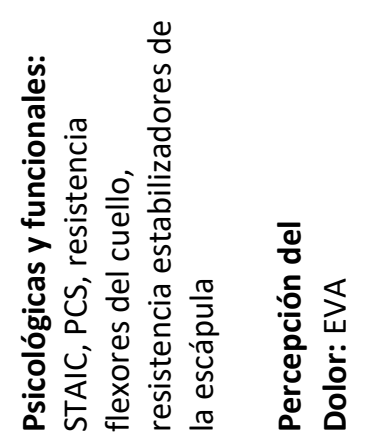

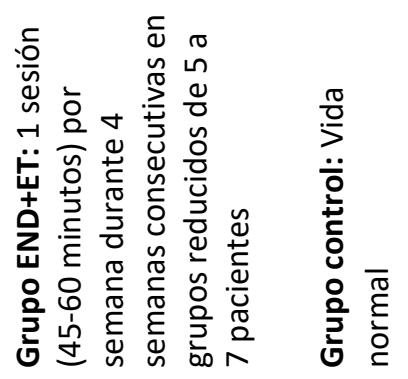

葶紊

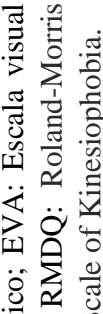

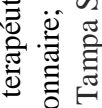

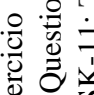

空的

$\ddot{\oplus}$

됭

$\frac{3}{9} \frac{3}{4}$

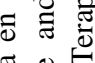

药

$>$

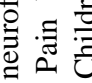

бо $\ddot{0}$

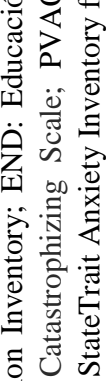

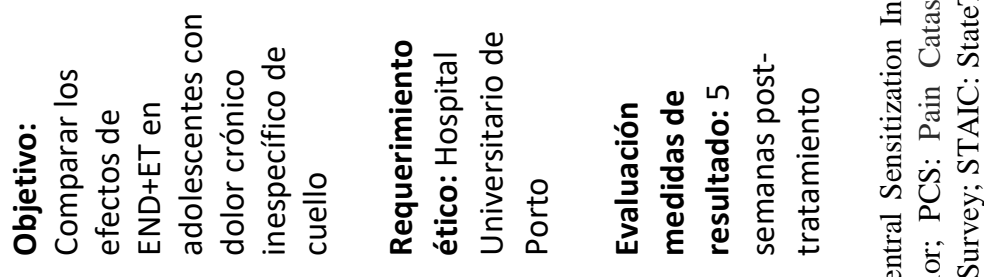

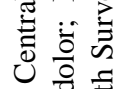

剀导

论 :

总氙

幽艺

๘

焉空完

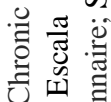

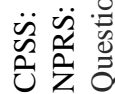




\section{DISCUSIÓN}

El objetivo de esta revisión bibliográfica fue evaluar la evidencia científica actual del empleo en conjunto de la educación en neurociencia del dolor y el ejercicio terapéutico en relación con la intensidad del dolor y las variables psicosociales en pacientes con dolor. Esta revisión sugiere que los pacientes que siguieron el tratamiento combinado de educación
También parece la combinación de educación en neurociencia del dolor con otras terapias convencionales como la punción seca o la terapia manual, parecen indicar una mejor evolución del dolor y una mejora significativa de la kinesofobia, la ansiedad y la actitud hacia el dolor cuando se compara con un tratamiento único de punción seca o terapia manual (Valiente-Castrillo et al., 2021).

El ejercicio físico podría ser una herramienta útil

Tabla 2. Evaluación de la calidad metodológica basado en la escala PEDro.

\begin{tabular}{|c|c|c|c|c|c|c|c|c|c|c|c|}
\hline & 1 & 2 & 3 & 4 & 5 & 6 & 7 & 8 & 9 & 10 & Total \\
\hline Galán-Martín et al. 2020 & 1 & 1 & 1 & 1 & 0 & 0 & 0 & 1 & 1 & 1 & 6 \\
\hline Andias et al. 2018 & 1 & 1 & 1 & 1 & 0 & 0 & 0 & 1 & 1 & 1 & 6 \\
\hline Bodes Pardo et al. 2018 & 1 & 1 & 1 & 1 & 0 & 0 & 1 & 1 & 1 & 1 & 7 \\
\hline López et al. 2018 & 1 & 1 & 1 & 1 & 0 & 0 & 0 & 1 & 1 & 1 & 6 \\
\hline Grande-Alonso et al. 2019 & 1 & 1 & 1 & 1 & 0 & 0 & 1 & 1 & 1 & 1 & 7 \\
\hline Malfliet et al. 2018 & 1 & 1 & 1 & 1 & 0 & 0 & 1 & 1 & 1 & 1 & 7 \\
\hline
\end{tabular}

1: se especifican los criterios de selección de sujetos; 2: se especifican los criterios de asignación aleatoria de sujetos a los grupos; 3 : se asignan al azar los sujetos a los grupos; 4: los grupos eran similares al inicio; 5: todos los sujetos estaban cegados; 6: todos los terapeutas estaban cegados; 7: todos los evaluadores estaban cegados; 8: se obtuvieron medidas de al menos uno de los resultados clave a partir de más del 85\% de los sujetos de la línea de base; 9: se realizó el análisis del tipo intención de tratar ; 10: se informaron los resultados de las comparaciones estadísticas entre los grupos en por lo menos para uno de los resultados clave; 11: el estudio proporciona medidas de la puntuación y de la variabilidad de, al menos, para uno de los resultados clave.

neurocientífica y ejercicio terapéutico obtuvieron resultados positivos más significativos que los producidos por otras terapias empleadas en los grupos de control. Un hallazgo importante es la mejor evolución del dolor en los grupos experimentales, posiblemente debido a los mejores resultados en las variables psicosociales que produce el tratamiento con educación neurocientífica.

La mejora de las variables cognitivo-emocionales podría lograrse porque la educación neurocientífica produce una reconceptualización y un cambio de actitud hacia el dolor que desincentiva los mecanismos centrales del procesamiento nociceptivo que provocan respuesta hipersensible (Nijs et al., 2016; Malfliet et al., 2018). De hecho, parece, que la educación neurocientífica como única terapia obtiene resultados favorables en los pacientes con niveles elevados de catastrofismo, kinesofobia e hipervigilancia (Malfliet et al., 2018). en la ayuda de la producción de analgesia y la modulación del dolor, pero sin embargo podría ser menos efectivo si no se incluye dentro de un contexto neurocientífico como sugieren los artículos analizados en esta revisión (Rice et al., 2019; GalanMartin et al., 2020). Una posible causa de porque los grupos experimentales analizados obtienen mejores resultados que los grupos control puede deberse al vínculo entre la kinesiofobia y el ejercicio terapéutico (Vlaeyen et al., 2002; Meulders, 2020). En este sentido, parece que la kinesofobia es la variable que mejor pronostico tiene con el uso de la educación neurocientífica. Desde este enfoque, el ejercicio terapéutico es una forma de exponer gradualmente en intensidad y dificultad al paciente hacia los gestos que le producen alerta y desencadenan una respuesta dolorosa. Por lo tanto, incluir el ejercicio gradual como terapia podría desarrollar una mejor comprensión durante el proceso de educación que desemboque en un descenso mayor del dolor. 


\section{Limitaciones}

La presente revisión sistemática presenta algunas limitaciones que es necesario tener en cuenta en la interpretación de los resultados. En primer lugar, es necesario destacar la falta de literatura científica en este campo, así como la heterogeneidad entre las formas de tratamiento y evaluación de los estudios incluidos, lo que limita la capacidad de extraer conclusiones sólidas. En segundo lugar, el diseño de los estudios y la calidad metodológica de los mismos son deficientes en muchos de los casos y presentan carencias que es necesario considerar en la generalización de los hallazgos encontrados. Por último, aunque se siguió un procedimiento sistemático, el riesgo de selección en los estudios sigue estando presente y es necesario confirmar estos hallazgos en futuras investigaciones.

\section{CONCLUSIÓN}

La terapia conjunta de educación en neurofisiología del dolor y el ejercicio terapéutico produce una mejora en las variables de ámbito psicosocial y las variables sensibles al dolor cuando es comparada con el ejercicio gradual o propuestas basadas en estrategias pasivas. El ejercicio implementado desde el punto de vista bioconductual parece ser más beneficioso que el ejercicio de fisioterapia convencional en pacientes con dolor crónico, aunque son necesarios futuros estudios que confirmen estos hallazgos y que faciliten su traslado a la práctica clínica de forma eficaz.

\section{FRASES DESTACADAS}

$>$ La combinación de educación y ejercicio terapéutico parece efectiva en la disminución del dolor y mejora de factores psicosociales en pacientes con dolor crónico.

$>$ La reconceptualización de aspectos cognitivo-emocionales relacionados con el dolor, gracias a la educación, permite influir sobre los mecanismos del procesamiento nociceptivo.

\section{REFERENCIAS}

Adams LM, Turk DC. Central sensitization and the biopsychosocial approach to understanding pain. J Appl Biobehav Res. John Wiley \& Sons, Ltd; 2018;23(2):e12125 DOI: http://dx.doi.org/10.1111/JABR.12125.

Andias R, Neto M, Silva AG. The effects of pain neuroscience education and exercise on pain, muscle endurance, catastrophizing and anxiety in adolescents with chronic idiopathic neck pain: a school-based pilot, randomized and controlled study. Physiother Theory Pract. Physiother Theory Pract; 2018;34(9):682-91 DOI: http://dx.doi.org/10.1080/09593985.2018.1423590.

Bodes Pardo G, Lluch Girbés E, Roussel N, Gallego Izquierdo T, Jiménez Penick V, Martín Pecos D. Pain Neurophysiology Education and Therapeutic Exercise for Patients With Chronic Low Back Pain: A Single-Blind Randomized Controlled Trial. Arch Phys Med Rehabil. Arch Phys Med Rehabil; 2018;99(2):338-47 DOI: http://dx.doi.org/10.1016/J.APMR.2017.10.016.

Booth J, Moseley G, Schiltenwolf M, Cashin A, Davies M, Hübscher M. Exercise for chronic musculoskeletal pain: A biopsychosocial approach. Musculoskeletal Care. Musculoskeletal Care; 2017;15(4):413-21 DOI: http://dx.doi.org/10.1002/MSC.1191.

Ceballos-Laita L, Mingo-Gómez MT, Navas-Cámara F, Estébanez-de-Miguel E, Caudevilla-Polo S, Verde-Rello Z, Fernández-Araque A, Jiménez-del-Barrio S. Therapeutic Exercise and Pain Neurophysiology Education in Female Patients with Fibromyalgia Syndrome: A Feasibility Study. J Clin Med. MDPI AG; 2020;9(11):3564 DOI: http://dx.doi.org/10.3390/jcm9113564.

Cuenca-Martínez F, Cortés-Amador S, Espí-López G. Effectiveness of classic physical therapy proposals for chronic non-specific low back pain: a literature review. Phys Ther Res. Phys Ther Res; 2018;21(1):16-22 DOI: http://dx.doi.org/10.1298/PTR.E9937.

Galan-Martin M, Montero-Cuadrado F, Lluch-Girbes E, CocaLópez M, Mayo-Iscar A, Cuesta-Vargas A. Pain Neuroscience Education and Physical Therapeutic Exercise for Patients with Chronic Spinal Pain in Spanish Physiotherapy Primary Care: A Pragmatic Randomized Controlled Trial. J Clin Med. J Clin Med; 2020;9(4):1201 DOI: http://dx.doi.org/10.3390/JCM9041201.

Grande-Alonso M, Suso-Martí S, Cuenca-Martínez F, PardoMontero J, Gil-Martínez A, La Touche R. Physiotherapy Based on a Biobehavioral Approach with or Without Orthopedic Manual Physical Therapy in the Treatment of Nonspecific Chronic Low Back Pain: A Randomized Controlled Trial. Pain Med. Pain Med; 2019;20(12):257187 DOI: http://dx.doi.org/10.1093/PM/PNZ093.

Koltyn, Brellenthin AG, Cook DB, Sehgal N, Hillard C. Mechanisms of Exercise-Induced Hypoalgesia. J Pain. 2014;15(12):1294-304

DOI: 
http://dx.doi.org/10.1016/j.jpain.2014.09.006.

Langley PC. The prevalence, correlates and treatment of pain in the European Union. Curr Med Res Opin. Curr Med Res Opin; 2011;27(2):463-80 DOI: http://dx.doi.org/10.1185/03007995.2010.542136.

Liberati A, Altman DG, Tetzlaff J, Mulrow C, Gøtzsche PC, Ioannidis JPA, Clarke M, Devereaux PJ, Kleijnen J, Moher D. The PRISMA statement for reporting systematic reviews and meta-analyses of studies that evaluate health care interventions: explanation and elaboration. Ann Intern Med. 2009;151(4):W65-94.

Louw A, Diener I, Butler DS, Puentedura EJ. The effect of neuroscience education on pain, disability, anxiety, and stress in chronic musculoskeletal pain. Arch Phys Med Rehabil. United States; 2011;92(12):2041-56 DOI: http://dx.doi.org/10.1016/j.apmr.2011.07.198.

Maher CG, Sherrington C, Herbert RD, Moseley AM, Elkins M. Reliability of the PEDro Scale for Rating Quality of Randomized Controlled Trials. Phys Ther. Oxford Academic; 2003;83(8):713-21 DOI: http://dx.doi.org/10.1093/PTJ/83.8.713.

Malfliet A, Kregel J, Coppieters I, De Pauw R, Meeus M, Roussel N, Cagnie B, Danneels L, Nijs J. Effect of Pain Neuroscience Education Combined With CognitionTargeted Motor Control Training on Chronic Spinal Pain: A Randomized Clinical Trial. JAMA Neurol. JAMA Neurol; 2018a;75(7):808-17 DOI: http://dx.doi.org/10.1001/JAMANEUROL.2018.0492.

Malfliet A, Kregel J, Meeus M, Danneels L, Cagnie B, Roussel N, Nijs J. Patients With Chronic Spinal Pain Benefit From Pain Neuroscience Education Regardless the Self-Reported Signs of Central Sensitization: Secondary Analysis of a Randomized Controlled Multicenter Trial. PM\&R. John Wiley \& Sons, Ltd; 2018b;10(12):1330-1343.e1 DOI: http://dx.doi.org/10.1016/J.PMRJ.2018.04.010.

Meulders A. Fear in the context of pain: Lessons learned from 100 years of fear conditioning research. Behav Res Ther. Elsevier Ltd; 2020;131:103635 DOI: http://dx.doi.org/10.1016/j.brat.2020.103635.

Moseley GL, Flor H. Targeting Cortical Representations in the Treatment of Chronic Pain: A Review. Neurorehabil Neural Repair. 2012;26(6):646-52 DOI: http://dx.doi.org/10.1177/1545968311433209.

Nijs J, Goubert D, Ickmans I. Recognition and Treatment of Central Sensitization in Chronic Pain Patients: Not Limited to Specialized Care. J Orthop Sports Phys Ther. J Orthop Sports Phys Ther; 2016;46(12):1024-8 DOI: http://dx.doi.org/10.2519/JOSPT.2016.0612.

Pergolizzi J, Ahlbeck K, Aldington D, Alon E, Coluzzi F, Dahan A, Huygen F, Kocot-Kępska M, Mangas AC, Mavrocordatos P, Morlion B, Müller-Schwefe G, Nicolaou A, Pérez Hernández C, Sichère P, Schäfer M, Varrassi G. The development of chronic pain: physiological CHANGE necessitates a multidisciplinary approach to treatment. Curr Med Res Opin. Taylor \& Francis; 2013;29(9):1127-35 DOI: http://dx.doi.org/10.1185/03007995.2013.810615.
Raja SN, Carr DB, Cohen M, Finnerup NB, Flor H, Gibson S, Keefe FJ, Mogil JS, Ringkamp M, Sluka KA, Song X-J, Stevens B, Sullivan MD, Tutelman PR, Ushida T, Vader K. The revised International Association for the Study of Pain definition of pain: concepts, challenges, and compromises. Pain. Ovid Technologies (Wolters Kluwer Health); 2020;161(9):1976-82 DOI: http://dx.doi.org/10.1097/j.pain.0000000000001939.

Rice D, Nijs J, Kosek E, Wideman T, Hasenbring MI, Koltyn K, Graven-Nielsen T, Polli A. Exercise-Induced Hypoalgesia in Pain-Free and Chronic Pain Populations: State of the Art and Future Directions. J Pain. United States; 2019;20(11):1249-66 http://dx.doi.org/10.1016/j.jpain.2019.03.005.

Roussel NA, Nijs J, Meeus M, Mylius V, Fayt C, Oostendorp R. Central Sensitization and Altered Central Pain Processing in Chronic Low Back Pain. Clin J Pain. 2013;29(7):625-38 DOI: http://dx.doi.org/10.1097/AJP.0b013e31826f9a71.

Stone PW. Popping the (PICO) question in research and evidence-based practice. Appl Nurs Res. 2002;15(3):197-8 DOI: http://dx.doi.org/10.1053/apnr.2002.34181.

Turk DC, Fillingim RB, Ohrbach R, Patel K V. Assessment of Psychosocial and Functional Impact of Chronic Pain. J Pain. Churchill Livingstone Inc.; 2016;17(9):T21-49 DOI: http://dx.doi.org/10.1016/j.jpain.2016.02.006.

Vaegter HB, Jones MD. Exercise-induced hypoalgesia after acute and regular exercise: experimental and clinical manifestations and possible mechanisms in individuals with and without pain. PAIN Reports. Ovid Technologies (Wolters Kluwer Health); 2020;5(5):e823 DOI: http://dx.doi.org/10.1097/PR9.0000000000000823.

Valiente-Castrillo P, Martín-Pintado-Zugasti A, Calvo-Lobo C, Beltran-Alacreu H, Fernández-Carnero J. Effects of pain neuroscience education and dry needling for the management of patients with chronic myofascial neck pain: a randomized clinical trial. Acupunct Med. Acupunct Med; 2021;39(2):91-105

DOI: http://dx.doi.org/10.1177/0964528420920300.

Verhagen AP, Vet HCW de, Bie RA de, Kessels AGH, Boers M, Bouter LM, Knipschild PG. The Delphi List: A Criteria List for Quality Assessment of Randomized Clinical Trials for Conducting Systematic Reviews Developed by Delphi Consensus. J Clin Epidemiol. Elsevier; 1998;51(12):123541 DOI: http://dx.doi.org/10.1016/S0895-4356(98)00131-0.

Vlaeyen JWS, De Jong J, Geilen M, Heuts PHTG, Van Breukelen G. The treatment of fear of movement/(re)injury in chronic low back pain: Further evidence on the effectiveness of exposure in vivo. Clin J Pain. Clin J Pain; 2002;18(4):251-61 DOI: http://dx.doi.org/10.1097/00002508-200207000-00006. 